\title{
RECTIFIABLE METRIC SPACES: LOCAL STRUCTURE AND REGULARITY OF THE HAUSDORFF MEASURE
}

\author{
BERND KIRCHHEIM
}

(Communicated by Andrew Bruckner)

\begin{abstract}
We consider the question whether the "nice" density behaviour of Hausdorff measure on rectifiable subsets of Euclidian spaces preserves also in the general metric case. For this purpose we show the existence of a metric differential of Lipschitzian functions also in situations where the well-known theorem of Rademacher fails.
\end{abstract}

Let $(X, \rho)$ be a metric space. We denote by $\mathscr{H}_{\rho}^{n}$ the $n$-dimensional Hausdorff measure over $X$ defined by

$$
\begin{aligned}
\mathscr{H}^{n}(E) & =\mathscr{H}_{\rho}^{n}(E) \\
& =\liminf _{\delta \searrow 0}\left\{\sum_{i=1}^{\infty} \frac{\alpha(n)}{2^{n}}\left(\operatorname{diam}_{\rho}\left(E_{i}\right)\right)^{n} \mid E \subset \bigcup_{i} E_{i}, \operatorname{diam}_{\rho}\left(E_{i}\right)<\delta\right\},
\end{aligned}
$$

where $\alpha(n)=\Gamma(1 / 2)^{n} / \Gamma(n / 2+1)$ is the volume of the unit ball in $\mathbb{R}^{n}$, and we say that $A \subset(X, \rho)$ is $n$-rectifiable if there are countably many Lipschitzian mappings $f_{i}, i \geq 1$, defined on subsets of $\mathbb{R}^{n}$ such that $\mathscr{H}_{\rho}^{n}\left(A \backslash \bigcup_{i} \operatorname{imag} f_{i}\right)=0$.

It is well known (see, e.g., [4, Chapters 3.2-3.3]), that $n$-rectifiable subsets of Euclidian spaces exhibit many properties similar to those of $n$-dimensional $\mathscr{C}^{1}$. submanifolds. For instance, if we denote by $B(x, r) \quad\left(=B_{\rho}(x, r)\right)$ the closed ball centered at $x$ of radius $r$, then for $\mathscr{H}^{n}$-almost every $x$ of an $n$-rectifiable $A \subset \mathbb{R}^{m}$ with $\mathscr{H}^{n}(A)<\infty$ we have $\lim _{r \backslash 0} \mathscr{H}^{n}(A \cap B(x, r)) / \alpha(n) r^{n}=1$. This means that the measure of small balls comes close to the volume of balls with equal radii in $\mathbb{R}^{n}$, and such points $x$ are called points of regularity of $\mathscr{H}^{n}$. The classical proof of this fact is based on Rademacher's theorem about almost everywhere differentiability of real Lipschitzian functions which allows the covering of the rectifiable set even by $\mathscr{C}^{1}$-pieces.

As any metric space isometrically embeds in some Banach space, we can suppose $X$ to have a (complete) norm $\|\cdot\|$ and will study Lipschitzian maps $f: \mathbb{R}^{n} \rightarrow(X,\|\cdot\|)$. Indeed, the assumption that $\operatorname{dmn} f=\mathbb{R}^{n}$ is no restriction since due to [5] any Lipschitzian $\tilde{f}: A \rightarrow(X,\|\cdot\|)$ has a Lipschitzian extension $f: \mathbb{R}^{n} \rightarrow(X,\|\cdot\|)$ (alternatively, embed $(X, \rho)$ into $\ell^{\infty}$ and

Received by the editors August 4, 1992.

1991 Mathematics Subject Classification. Primary 28A78; Secondary 26B10.

Key words and phrases. Rectifiability, metric differential, Hausdorff measure, density, Kolmogoroff measure. 
then the extension is found easily). It is well known that the statement that any such $f$ has the (Fréchet) differential almost everywhere is equivalent to the condition that $(X,\|\cdot\|)$ has the Radon-Nikodým property (see [1]) and, therefore, is far from being true generally. For instance, the isometric map $\Phi:[0,1] \rightarrow L^{1}([0,1])$ defined by $\Phi(t)=\chi_{[0, t]}$ has derivative at no point $x \in[0,1]$. We overcome this difficulty by proving a metrical version of the Rademacher theorem which shows that the "nice" metrical behaviour of the example $\Phi$ above is typical. Based on this result we can generalize in Lemma 4, Theorem 7, and Corollary 8 the approach from Chapter 3.2 of [4] to derive an area formula for general Banach space-valued Lipschitzian mappings. We can also prove the regularity almost everywhere of $\mathscr{H}^{n}$ on $n$-rectifiable metric spaces of finite Hausdorff measure. This question was the starting point for the considerations presented here.

Observe that in the Euclidian case regularity of the Hausdorff measure is not just a consequence but even a characterization of rectifiability; see [8] for the much more complicated converse implication. Moreover, recently the equivalence of these two notions for $n=1$ but in the general metric setting was established in [10]. So the natural question appears whether also for general $n$ this equivalence holds.

Proposition 1. Let $f: \mathbb{R}^{n} \rightarrow(X,\|\cdot\|)$ be Lipschitzian and $u \in \mathbb{S}^{n-1}$ arbitrary. Then for almost each $x \in \mathbb{R}^{n} \lim _{r \backslash 0}\|f(x+r u)-f(x)\| / r$ exists.

Proof. Since $f$ is continuous, the set $\mathscr{D}_{u}$ of all $x \in \mathbb{R}^{n}$, where the desired limit exists, is a Borel one. Therefore, by Fubini's theorem it suffices to prove $\mathscr{H}_{|\cdot|}^{1}\left(\left(y_{0}+\mathbb{R} \cdot u\right) \backslash \mathscr{D}_{u}\right)=0$ for any $y_{0} \in \mathbb{R}^{n}$. Here and in the sequel $|\cdot|$ denotes the Euclidian norm. Define on $\mathbb{R}$ the metric

$$
\rho(x, y)=|x-y|+\left\|f\left(y_{0}+x u\right)-f\left(y_{0}+y u\right)\right\| .
$$

Since $\rho$ and the Euclidian metric are bilipschitzianly equivalent, we see that $\mathscr{H}_{\rho}{ }^{1}$ is locally finite on $\mathbb{R}$ and that for $\mathscr{H}_{\rho}{ }^{1}$-almost every, i.e., for $\mathscr{L}^{1}$-a.e. $x \in \mathbb{R}$ the following holds (see, e.g., $[4,2.10 .18(3)])$ :

$$
\lim _{r \searrow 0} \sup \left\{\frac{\mathscr{H}_{\rho}^{1}(M)}{\operatorname{diam}_{\rho}(M)} \mid x \in M \subset[x-r, x+r]\right\}=1 .
$$

Next, since monotone functions on the real line are differentiable almost everywhere, we see that for $\mathscr{L}^{1}$-a.e. $x \in \mathbb{R}$

$$
\lim _{r \searrow 0} \frac{1}{r} \mathscr{H}_{\rho}^{1}([x, x+r]) \in[1, \infty)
$$

Obviously, the proposition follows if we show that $\lim _{r \backslash 0} \rho(x, x+r) / r$ exists whenever $x$ fulfills (1) and (2).

But then for any $r>0$ there are $a(r)<b(r)$ in $[x, x+r]$ with $\rho(a(r), b(r))$ $=\operatorname{diam}_{\rho}([x, x+r])$. We infer that

$$
\begin{aligned}
\mathscr{H}_{\rho}^{1}([x, x+r]) & =\mathscr{H}_{\rho}^{1}([x, a(r)])+\mathscr{H}_{\rho}^{1}([a(r), b(r)])+\mathscr{H}_{\rho}^{1}([b(r), x+r]) \\
& \geq \rho(a(r), b(r))+\rho(x, a(r))+\rho(b(r), x+r) \\
& \geq \operatorname{diam}_{\rho}([x, x+r])+\left(\operatorname{diam}_{\rho}([x, x+r])-\rho(x, x+r)\right) .
\end{aligned}
$$


Indeed, due to $[4,2.10 .12]$ set $\mathscr{H}_{\rho}^{1}(M) \geq \operatorname{diam}_{\rho}(M)$ for any $M$ connected and then use the triangle inequality. Hence, (1) implies

$$
1 \geq \underset{r \searrow 0}{\lim \sup _{0}} \frac{\mathscr{H}_{\rho}^{1}([x, x+r])}{\operatorname{diam}_{\rho}([x, x+r])} \geq \underset{r \searrow 0}{\lim \sup }\left(1+\left|\frac{\rho(x, x+r)}{\operatorname{diam}_{\rho}([x, x+r])}-1\right|\right) .
$$

Therefore,

$\lim _{r \searrow 0} \rho(x, x+r) / \operatorname{diam}_{\rho}([x, x+r])=\lim _{r \searrow 0} \mathscr{H}_{\rho}^{1}([x, x+r]) / \operatorname{diam}_{\rho}([x, x+r])=1$, and now (2) finishes the proof.

We shall denote

$$
M D(f, x)(u)=\lim _{r \searrow 0} \frac{1}{r}\|f(x+r u)-f(x)\| \quad \text { for } x, u \in \mathbb{R}^{n}
$$

whenever this limit exists. Obviously, in this case

$$
M D(f, x)(\lambda u)=\lambda M D(f, x)(u) \text { for any } \lambda \geq 0 .
$$

Theorem 2. Let $f: \mathbb{R}^{n} \rightarrow(X,\|\cdot\|)$ be Lipschitzian. Then, for almost each $x \in \mathbb{R}^{n}, M D(f, x)(\cdot)$ is a seminorm on $\mathbb{R}^{n}$ and

$$
\|f(z)-f(y)\|-M D(f, x)(z-y)=o(|z-x|+|y-x|) .
$$

Proof. Let $\left\{u_{m}\right\}_{m=1}^{\infty}$ be dense in $\mathbb{S}^{n-1}$. According to the foregoing Proposition 1 for almost each $x \in \mathbb{R}^{n}$ and any $m \geq 1 M D(f, x)\left(u_{m}\right)$ exists finitely. Using Egoroff's theorem and the Lebesgue Density theorem, one easily sees that it suffices to prove the assertion about $M D\left(f, x_{0}\right)$ provided that $x_{0}$ is a (Lebesgue) density point of a compact set $K \subset \mathbb{R}^{n}$ such that for each $\varepsilon>0$ and $m \geq 1$ there is an $r(\varepsilon, m)>0$ with

$$
\left|\frac{1}{r}\|f(x)-f(x+r u)\|-M D(f, x)(u)\right| \leq \varepsilon .
$$

on $K \times\left\{u_{k}\right\}_{k=1}^{m}$ whenever $r \in(0, r(\varepsilon, m))$.

First, using the Lipschitz continuity of $f$ one observes that $M D(f, x)(u)$ is defined for any $x \in K$ and $\operatorname{Lip}(f)$-Lipschitzian on the entire sphere $\mathbb{S}^{n-1}$. Moreover, for any $\varepsilon>0$ there is an $r_{\varepsilon}>0$ such that (4) holds on $K \times \mathbb{S}^{n-1}$ if $r \in\left(0, r_{\varepsilon}\right)$. Indeed, for $m \geq 1, v \in\left\{u_{k} \mid k \leq m\right\}, u \in \mathbb{S}^{n-1}$, and arbitrary $r \in(0, r(\varepsilon, m))$ we have

$$
\left|\frac{1}{r}\|f(x+r u)-f(x)\|-M D(f, x)(v)\right| \leq \varepsilon+\operatorname{Lip}(f)|u-v|,
$$

which gives the existence of $M D(f, x)(u)$ and the estimate

$$
\begin{aligned}
& \left|\frac{1}{r}\|f(x+r u)-f(x)\|-M D(f, x)(u)\right| \\
& \quad \leq 2 \varepsilon+2 \operatorname{Lip}(f) \operatorname{dist}\left(u,\left\{u_{k} \mid 1 \leq k \leq m\right\}\right) .
\end{aligned}
$$

Next, we claim that

$$
\lim _{r \searrow 0} \frac{1}{r}\left\|f\left(x_{0}+r x\right)-f\left(x_{0}+r y\right)\right\|=M D\left(f, x_{0}\right)(x-y)
$$

locally uniformly in $x, y \in \mathbb{R}^{n}$. For given $\varepsilon>0$ we fix $\delta>0$ such that

- $B\left(x_{0}+r y, \varepsilon r\right) \cap K \neq \varnothing$ for $|y| \leq \frac{1}{\varepsilon}$ and $r \in(0, \delta) \quad\left(x_{0}\right.$ density point); 
- $\left|\frac{1}{r}\|f(x+r u)-f(x)\|-M D(f, x)(u)\right|<\varepsilon^{2}$ if $x \in K, u \in \mathbb{S}^{n-1}$, $r<2 \delta / \varepsilon$

- $\left|M D\left(f, x_{0}\right)(u)-M D(f, y)(u)\right|<\varepsilon^{2}$ if $u \in \mathbb{S}^{n-1}$ and $y \in K \cap$ $B\left(x_{0}, \delta\left(\varepsilon+\frac{1}{\varepsilon}\right)\right)$.

Note that due to (4) $M D(f, \cdot)(\cdot)$ is continuous on $K \times \mathbb{S}^{n-1}$. Now, for arbitrary $x, y \in B\left(0, \frac{1}{\varepsilon}\right)$ and $r \in(0, \delta)$ we find $z \in K \cap B\left(x_{0}+r y, \varepsilon r\right) \subset$ $B\left(x_{0}, \delta\left(\varepsilon+\frac{1}{\varepsilon}\right)\right)$ and can estimate

$$
\begin{aligned}
& \left|\frac{1}{r}\left\|f\left(x_{0}+r x\right)-f\left(x_{0}+r y\right)\right\|-M D\left(f, x_{0}\right)(x-y)\right| \\
& \leq\left|\frac{1}{r}\|f(z+r(x-y))-f(z)\|-M D\left(f, x_{0}\right)(x-y)\right| \\
& \quad+\frac{1}{r}\left\|f\left(x_{0}+r x\right)-f(z+r(x-y))\right\|+\frac{1}{r}\left\|f\left(x_{0}+r y\right)-f(z)\right\| \\
& \leq|x-y|\left(\left|\frac{1}{r|x-y|}\right|\left|f\left(z+r|x-y| \frac{x-y}{|x-y|}\right)-f(z) \|-M D(f, z)\left[\frac{x-y}{|x-y|}\right]\right|\right. \\
& \left.\quad+\left|M D\left(f, x_{0}\right)\left[\frac{x-y}{|x-y|}\right]-M D(f, z)\left[\frac{x-y}{|x-y|}\right]\right|\right) \\
& \quad+\frac{2}{r}\left(\operatorname{Lip}(f)\left|x_{0}+r y-z\right|\right) \\
& \leq \frac{2}{\varepsilon}\left(\varepsilon^{2}+\varepsilon^{2}\right)+\operatorname{Lip}(f)\left(\frac{2}{r} \cdot \varepsilon r\right) \leq(4+2 \operatorname{Lip}(f)) \varepsilon,
\end{aligned}
$$

which proves the claim (the case $x=y$ being trivial).

We finish the proof by showing that $M D\left(f, x_{0}\right)$ is a seminorm fulfilling (3). From (5) we infer $M D\left(f, x_{0}\right)(y)+M D\left(f, x_{0}\right)(z) \geq M D\left(f, x_{0}\right)(y+z)$ and $M D\left(f, x_{0}\right)(y)=M D\left(f, x_{0}\right)(-y)$; hence, we have a seminorm. Moreover, for $\varepsilon>0$ we find $\delta_{\varepsilon}>0$ with

$$
\left|\frac{1}{r}\left\|f\left(x_{0}+r y\right)-f\left(x_{0}+r z\right)\right\|-M D\left(f, x_{0}\right)(y-z)\right| \leq \varepsilon
$$

for $|y|,|z| \leq \frac{1}{\varepsilon}$ and $r \in\left(0,2 \delta_{\varepsilon}\right)$. So, if $\varepsilon<1$ and $|\widetilde{y}|,|\widetilde{z}| \leq \delta_{\varepsilon}$ then for $r=|\tilde{y}|+|\tilde{z}|$ and $y=\tilde{y} / r, z=\tilde{z} / r \in B\left(0, \frac{1}{\varepsilon}\right)$ we obtain

$$
\left|\left\|f\left(x_{0}+\tilde{y}\right)-f\left(x_{0}+\widetilde{z}\right)\right\|-M D\left(f, x_{0}\right)(r(y-z))\right| \leq \varepsilon r \leq \varepsilon(|\tilde{y}|+|\widetilde{z}|),
$$

which is just (3).

Definition 3. Let $f: \mathbb{R}^{n} \rightarrow(X,\|\cdot\|)$ be a mapping and $x \in \mathbb{R}^{n}$. In case that there is a seminorm $s$ on $\mathbb{R}^{n}$ satisfying (3), we call $s$ the metric differential of $f$ at $x$.

Of course, in case such an $s$ exists it is unique and equal to $M D(f, x)$. Moreover, if the Fréchet differential $D f(x)$ exists then $M D(f, x)(u)=$ $\|D f(x)(u)\|$.

Notation. For $f: \mathbb{R}^{n} \rightarrow(X,\|\cdot\|)$ Lipschitzian we denote by $\mathscr{M D}(f)$ the set of all $x$ where the metric differential exists and by $\mathscr{M D}_{r}(f)$ the subset of $\mathscr{M D}(f)$ where it is a norm on $\mathbb{R}^{n}$.

Using ideas from the proof of Theorem 2 it is quite easy to see that $\mathscr{M D}(f)$ as well as $\mathscr{M D}_{r}(f)$ are Borel sets. 
Lemma 4. Let $f: \mathbb{R}^{n} \rightarrow(X,\|\cdot\|)$ be Lipschitzian and $\lambda>1$. Then there are Borel subsets $\left\{E_{i}\right\}_{i \geq 1}$ of $\mathbb{R}^{n}$ and norms $\left\{\|\cdot\|_{i}\right\}_{i \geq 1}$ on $\mathbb{R}^{n}$ such that

(i) $\bigcup_{i=1}^{\infty} E_{i}=\mathscr{M D} \mathscr{D}_{r}(f)$ and

(ii) $\frac{1}{\lambda}\|x-y\|_{i} \leq\|f(x)-f(y)\|_{x} \leq \lambda\|x-y\|_{i}$ for $x, y \in E_{i}$.

Proof. First, observe that there is a countable dense subset $S$ in the space of all norms on $\mathbb{R}^{n}$ in the sense that for any norm $\|\cdot\|^{\prime}$ on $\mathbb{R}^{n}$ and any $\varepsilon>0$ we find a $\|\cdot\|^{\prime \prime} \in S$ with $(1-\varepsilon)\|x\|^{\prime \prime} \leq\|x\|^{\prime} \leq(1+\varepsilon)\|x\|^{\prime \prime}$ for any $x \in \mathbb{R}^{n}$. This follows, for example, from the separability of the space of all compact, convex, symmetrical bodies equipped with the Hausdorff metric (see [4, 2.10.21]). Now, the lemma can be established by following the proof of Lemma 3.2.2 in [4].

Definition 5. Let $s$ be a seminorm on $\mathbb{R}^{n}$. We define the "Jacobian of $s$ " by $\mathscr{J}(s)=\alpha(n) n\left(\int_{\mathbf{S}^{n-1}}[s(x)]^{-n} d \mathscr{H}^{n-1}(x)\right)^{-1}$.

Lemma 6. Let $\|\cdot\|$ be a norm on $\mathbb{R}^{n}$; then

(i) $\mathscr{K}_{\|\cdot\|}^{n}\left(B_{\|\cdot\| \|}(0,1)\right)=\alpha(n)$, and

(ii) for any $A \subset \mathbb{R}^{n}, \mathscr{L}_{\|\cdot\|}^{n}(A)=\mathscr{J}(\|\cdot\|) \mathscr{L}^{n}(A)$.

Proof. Since id $\left(\mathbb{R}^{n},|\cdot|\right) \rightarrow\left(\mathbb{R}^{n},\|\cdot\|\right)$ is a bilipschitzian mapping, $\mathscr{H}_{\|\cdot\|}^{n}$ is a locally finite nontrivial measure. Because of its translation invariance, there is a constant $c>0$ with $\mathscr{H}_{\|\cdot\|}^{n}=c \cdot \mathscr{L}^{n}$. To prove (i), we first observe that $\mathscr{H}_{\|\cdot\|}^{n}\left(B_{\|\cdot\|}(0,1)\right) \leq \alpha(n)$ due to the upper density estimate

$$
\underset{r \searrow 0}{\limsup } \mathscr{K}_{\|\cdot\|}^{n}\left(B_{\|\cdot\|}(x, r)\right) /\left(\alpha(n) r^{n}\right) \leq 1 \text { for } \mathscr{H}_{\|\cdot\|}^{n} \text {-a.e. } x
$$

(see $[4,2.10 .19(5)])$, and due to the "homogeneity" of the measure

$$
\mathscr{H}_{\|\cdot\|}^{n}(x+t \cdot A)=t^{n} \mathscr{X}_{\|\cdot\|}^{n}(A) \text { for } t \geq 0, x \in \mathbb{R}^{n} .
$$

For the converse inequality we use the general isodiametric inequality of Minkowskian geometry (see [3, Theorem 11.2.1]). It says that, for any $A \subset \mathbb{R}^{n}$, $\mathscr{L}^{n}(A) \leq \mathscr{L}^{n}\left(B_{\|\cdot\|}\left(0, \frac{1}{2} \operatorname{diam}_{\|\cdot\|}(A)\right)\right)$ and implies that any covering $\left\{A_{i}\right\}_{i=1}^{\infty}$ of $B_{\|\cdot\|}(0,1)$ fulfills the inequality $1 \leq \sum_{i=1}^{\infty}\left(\frac{1}{2} \operatorname{diam}_{\|\cdot\|}\left(A_{i}\right)\right)^{n}$. Hence, (i) follows.

To prove (ii) we compute using polar coordinates

$$
\begin{aligned}
\mathscr{L}^{n}\left(B_{\|\cdot\|}(0,1)\right) & =\int_{\mathbf{S}^{n-1}} \int_{0}^{\infty} r^{n-1} \chi_{B_{\|\bullet\|}(0,1)}(r x) d r d \mathscr{P}^{n-1}(x) \\
& =\int_{\mathbb{S}^{n-1}} \int_{0}^{1 /\|x\|} r^{n-1} d r d \mathscr{P}^{n-1}(x)=\frac{1}{n} \int_{\mathbf{S}^{n-1}}\|x\|^{-n} d \mathscr{H}^{n-1}(x)
\end{aligned}
$$

and $\mathscr{J}(\|\cdot\|)=c=\mathscr{H}_{\|\cdot\|}^{n}\left(B_{\|\cdot\|}(0,1)\right) / \mathscr{L}^{n}\left(B_{\|\cdot\|}(0,1)\right)$ follows.

Theorem 7. Let $f: \mathbb{R}^{n} \rightarrow(X,\|\cdot\|)$ be Lipschitzian, and let $A \subset \mathbb{R}^{n}$ be Lebesgue measurable. Then

$$
\int_{A} \mathscr{J}(M D(f, x)) d \mathscr{L}^{n}(x)=\int_{X} N(f \mid A, x) d \mathscr{H}_{\|\cdot\|}^{n}(x),
$$

where $N(f \mid A, x)$ denotes the cardinality of the set $A \cap f^{-1}(x)$.

Proof. First, we suppose that $A$ is a Borel subset of $\mathscr{N D}_{r}(f)$. Routine arguments show that $x \rightarrow \mathscr{J}(M D(f, x))$ is a Borel function on $A$. Choose $\lambda>1$ 
and find disjoint sets $\left\{E_{i}\right\}_{i \geq 1}$ and norms $\left\{\|\cdot\|_{i}\right\}_{i \geq 1}$ according to Lemma 4. Then for each $i \geq 1 \quad \lambda^{-n} \mathscr{K}_{\|\cdot\|_{i}}\left(E_{i} \cap A\right) \leq \mathscr{K}_{\|\cdot\|}^{n}\left(f\left(E_{i} \cap A\right)\right) \leq \lambda^{n} \mathscr{Z}_{\|\cdot\|_{i}}\left(E_{i} \cap A\right)$ and $\lambda^{-1}\|\cdot\|_{i} \leq M D(f, x)(\cdot) \leq \lambda\|\cdot\|_{i}$ if $x \in \mathscr{M D}_{r}(f)$ is a density point of $E_{i}$. Consequently, by the definition of $\mathscr{J}(s)$

$$
\lambda^{-n} \mathscr{J}\left(\|\cdot\|_{i}\right) \leq \mathscr{J}(M D(f, x)) \leq \lambda^{n} \mathscr{J}\left(\|\cdot\|_{i}\right) \text { a.e. on } E_{i}
$$

and according to Lemma 6

$$
\begin{aligned}
\lambda^{-2 n} \int_{E_{i} \cap A} \mathcal{J}(M D(f, \cdot)) d \mathscr{L}^{n} & \leq \int_{X} N\left(f \mid E_{i} \cap A, \cdot\right) d \mathscr{L}_{\|\cdot\|}^{n} \\
& \leq \lambda^{2 n} \int_{E_{i} \cap A} \mathcal{J}(M D(f, \cdot)) d \mathscr{L}^{n} .
\end{aligned}
$$

Summing over $i$ and declining with $\lambda$ to 1 gives the conclusion (6) in this first case.

Since obviously both sides of (6) equal zero if $A$ is a Lebesgue zero set, the theorem follows if we show that $\mathscr{J}(M D(f, x))=0$ for $x \in \mathscr{M D}(f) \backslash \mathscr{M D}{ }_{r}(f)$ and that $\mathscr{X}_{\|\cdot\|}^{n}(f(K))=0$ for any compact $K \subset \mathscr{M} \mathscr{D}(f) \backslash \mathscr{M O D}_{r}(f)$. We consider the product space $Y=X \times \mathbb{R}^{n}$ with the norm $\|(x, t)\|_{1}=\max \{\|x\|,|t|\}$ and the projection $\pi:(x, t) \rightarrow x$ and for any $\varepsilon>0$ define $f_{\varepsilon}(t)=(f(t), \varepsilon t)$. Since $\pi$ is 1-Lipschitzian and $f=\pi \circ f_{\varepsilon}$ for any $\varepsilon>0$, it suffices to prove that $\mathscr{X}_{\|\cdot\|_{1}}\left(f_{\varepsilon}(K)\right) \rightarrow 0$ as $\varepsilon \rightarrow 0$. Because always $\mathscr{M D}_{r}\left(f_{\varepsilon}\right)=\mathscr{M} \mathscr{D}(f)$, according to the first case we are done if we show that

$$
\mathscr{J}\left(M D\left(f_{\varepsilon}, x\right)\right) \leq \frac{\alpha(n) n}{\alpha(n-1)(n-1)}(\operatorname{Lip}(f))^{n-1} \cdot \varepsilon
$$

for any $x \in \mathscr{M D}(f) \backslash \mathscr{M D}_{r}(f)$ and $0<\varepsilon<\operatorname{Lip}(f) / 2$. Indeed, since

$$
M D(f, x)(\cdot) \leq M D\left(f_{\varepsilon}, x\right)(\cdot),
$$

we see also that the Jacobian of $f$ vanishes for such $x$. To prove (7), choose any $u \in \mathbb{S}^{n-1}$ with $M D(f, x)(u)=0$ and define the two mappings $p^{ \pm}(y)=$ $y \pm \sqrt{1-|y|^{2}} u$ on $C=B(0,1) \cap\{u\}^{\perp}$. Then $\mathbb{S}^{n-1}=p^{+}(C) \cup p^{-}(C)$, both $p^{+}, p^{-}$do not decrease distances, and, for any $y \in C$,

$$
M D(f, x)(y)=M D(f, x)\left(p^{+}(y)\right)=M D(f, x)\left(p^{-}(y)\right) .
$$

Because $M D\left(f_{\varepsilon}, x\right)(v)=\max \{M D(f, x)(v), \varepsilon|v|\}, v \in \mathbb{R}^{n}$, we can estimate

$$
\begin{aligned}
& \int_{\mathbf{S}^{n-1}}\left(M D\left(f_{\varepsilon}, x\right)(v)\right)^{-n} d \mathscr{H}^{n-1}(v) \\
& \geq 2 \int_{C}(\max \{M D(f, x)(y), \varepsilon\})^{-n} d \mathscr{H}^{n-1}(y) \\
& \geq 2 \int_{C} \min \left\{\varepsilon^{-n},((\operatorname{Lip}(f))|y|)^{-n}\right\} d \mathscr{C}^{n-1}(y) \\
& \geq 2(\operatorname{Lip}(f))^{-n} \int_{\varepsilon / \operatorname{Lip}(f) \leq|y| \leq 1}|y|^{-n} d \mathscr{C}^{n-1}(y) \\
& =2(\operatorname{Lip}(f))^{-n}(n-1) \alpha(n-1) \int_{\varepsilon / \operatorname{Lip}(f)}^{1} \frac{d t}{t^{2}} \\
& =\frac{2(\operatorname{Lip}(f)-\varepsilon)}{\varepsilon} \frac{(n-1) \alpha(n-1)}{(\operatorname{Lip}(f))^{n}},
\end{aligned}
$$


which gives together with Definition 5 for $\varepsilon<\frac{1}{2} \operatorname{Lip}(f)$ the desired inequality (7) and finishes the proof.

We obtain the following substitution rule for integration with respect to Hausdorff measure on rectifiable subsets of Banach spaces (see [4, Theorem 3.2.5] for weaker assumptions on the integrand).

Corollary 8 (area formula). Let $f: \mathbb{R}^{n} \rightarrow(X,\|\cdot\|)$ be Lipschitzian.

(i) If $g: \mathbb{R}^{n} \rightarrow \overline{\mathbb{R}}$ is Lebesgue integrable, then

$$
\int_{\mathbb{R}^{n}} g(x) \mathscr{J}(M D(f, x)) d \mathscr{L}^{n}(x)=\int_{X}\left(\sum_{x \in f^{-1}(y)} g(x)\right) d \mathscr{K}_{\|\cdot\|}^{n}(y) .
$$

(ii) If $g: X \rightarrow \overline{\mathbb{R}}$ is $\mathscr{H}_{\|\cdot\|}^{n}$-measurable, and $A \subset \mathbb{R}^{n}$ is $\mathscr{L}^{n}$-measurable, then

$$
\int_{A} g(f(x)) \mathscr{J}(M D(f, x)) d \mathscr{L}^{n}(x)=\int_{X} g(y) N(f \mid A, y) d \mathscr{H}_{\|\cdot\|}^{n}(y)
$$

provided at least one of the integrals exists.

The proof is based on Theorem 7 and standard approximation procedures.

Now we can describe the local structure of general rectifiable metric spaces.

Theorem 9. Let $(X, \rho)$ be n-rectifiable. Then for $\mathscr{H}_{\rho}^{n}$-a.e. $x \in X$ the following is true: There exist a norm $\|\cdot\|_{x}$ on $\mathbb{R}^{n}$, a map $\phi_{x}: X \rightarrow \mathbb{R}^{n}$, and a closed set $A_{x} \subset X$ such that $\phi_{x}(x)=0, \lim _{r \backslash 0}\left(\alpha(n) r^{n}\right)^{-1} \mathscr{H}_{\rho}^{n}\left(B_{\rho}(x, r) \cap A_{x}\right)=1$, and

$$
\lim _{r \searrow 0} \sup \left\{\left|1-\frac{\left\|\phi_{x}(y)-\phi_{x}(z)\right\|_{x}}{\rho(y, z)}\right| \mid y, z \in A_{x} \cap B_{\rho}(x, r) \text { different }\right\}=0 \text {. }
$$

Proof. First, we can suppose $(X, \rho)$ to be a metric subspace of a Banach space $(\tilde{X},\|\cdot\|)$; for the existence of such an isometric embedding see [4, 2.5.16]. Note that $\mathscr{H}_{\rho}^{n}(A)=\mathscr{H}_{\|\cdot\|}^{n}(A)$ for any $A \subset X$. From the definition of $n$ rectifiability and the already mentioned extension result [5] we infer that it suffices to prove that for any Lipschitzian $f: \mathbb{R}^{n} \rightarrow \widetilde{X}$ the conclusion holds at $\mathscr{K}_{\|\cdot\|^{n}}^{n}$-a.e. $x \in X \cap f(B(0,1))$. The outer regularity of $\mathscr{H}_{\|\cdot\|}^{n}$ ensures the existence of a $G_{\delta}$-set $X^{*}$ with $X \cap f(B(0,1)) \subset X^{*} \subset f(B(0,1))$ and $\mathscr{H}_{\|\cdot\|}^{n}\left(X^{*} \cap M\right)=$ $\mathscr{H}_{\rho}^{n}(X \cap f(B(0,1)) \cap M)$ for $M \subset \tilde{X}$ Borel. Put $S=f^{-1}\left(X^{*}\right) \cap B(0,1) \cap$ $\mathscr{M} \mathscr{D}_{r}(f)$ and $\lambda_{k}=1+2^{-k-1}$ for $k \geq 0$. Using Lemma 4 repeatedly we can find for $k=1,2, \ldots$ refining partitions $\left\{E_{i}^{k}\right\}_{i=1}^{\infty}$ of $S$ and norms $\left\{\|\cdot\|_{i}^{k}\right\}_{i=1}^{\infty}$ corresponding to $\lambda_{k}$. Denote by $G_{i}^{k}, i, k \geq 1$, the set of all $x \in E_{i}^{k}$ which are Lebesgue density points of $E_{i}^{k}$, and define $S^{*}=\bigcap_{k=1}^{\infty} \bigcup_{i=1}^{\infty} G_{i}^{k}$. Then $\mathscr{L}^{n}\left(S \backslash S^{*}\right)=0$, and $\mathscr{H}_{\|\cdot\|}^{n}\left(X^{*} \backslash f\left(S^{*}\right)\right)=0$ by Theorem 7. So, we are done if we show the conclusion for any $x=f(t)$ with $t \in S^{*}$. Since the $G_{i}^{k}$, $i, k \geq 1$, form a nested family, for any $k \geq 1$ we find the unique $G_{k}(t)=$ $G_{i(t, k)}^{k}$ containing $t$ and the corresponding norm $\|\cdot\|_{k}=\|\cdot\|_{i(t, k)}^{k}$. Because $G_{k}(t) \supset G_{k+1}(t)$ have density one at $t$ and $\lambda_{k+1} \lambda_{k} \leq \lambda_{k-1}$, we conclude from condition (ii) in Lemma 4 that $\lambda_{k-1}^{-1}\|v\|_{k} \leq\|v\|_{k^{\prime}} \leq \lambda_{k-1}\|v\|_{k}$ for $1 \leq k \leq k^{\prime}$ and $v \in \mathbb{R}^{n}$. Therefore, we can define a norm on $\mathbb{R}^{n}$ by $\|v\|_{x}=\lim _{k \rightarrow \infty}\|v\|_{k}$. 
Then for any $k \geq 1$

$$
\begin{aligned}
\left(1+2^{-k+1}\right)^{-1}\|y-z\|_{x} & \leq\|f(y)-f(z)\| \\
& \leq\left(1+2^{-k+1}\right)\|y-z\|_{x} \quad \text { if } y, z \in G_{k}(t) .
\end{aligned}
$$

Because $t$ is a density point for each $G_{k}(t)$, we find a sequence of radii $\left\{r_{k}\right\}_{k=1}^{\infty}$ with $0<r_{k+1}<r_{k} / 12 k$ such that $\mathscr{L}^{n}\left(G_{k}(t) \cap B(t, r)\right) \geq\left(1-\frac{1}{4 k}\right) \mathscr{L}^{n}(B(t, r))$ if $r \in\left[0, r_{k}\right]$. Denote $M_{k}=B\left(t, r_{k}\right) \backslash B\left(t, r_{k+1}\right)$, and one easily sees that for each $k \geq 1$ there is a closed set $A_{k} \subset G_{k}(t) \cap M_{k}$ such that $\mathscr{L}^{n}\left(A_{k}\right)>$ $\left(1-\frac{1}{3 k}\right) \mathscr{L}^{n}\left(M_{k}\right)$ and $\mathscr{L}^{n}\left(M_{k} \cap G_{k}(t) \backslash A_{k}\right)<\frac{1}{3 k} \mathscr{L}^{n}\left(B\left(t, r_{k+1}\right)\right)$. Then $A=$ $\bigcup_{k=1}^{\infty} A_{k} \cup\{t\}$ is a compact set,

$$
B\left(t, r_{k}\right) \cap A \subset G_{k}(t),
$$

and a straightforward but lengthy computation shows that

$$
\alpha(n) r^{n} \geq \mathscr{L}^{n}(A \cap B(t, r)) \geq\left(1-\frac{1}{k}\right) \alpha(n) r^{n} \quad \text { for } r \in\left(r_{k+1}, r_{k}\right]
$$

(compare with [7, Theorem 6.21]).

Finally, we define the set $A_{x}=f(A) \cap X$ closed in $X$ and the function

$$
\phi_{x}(y)= \begin{cases}p-t & \text { for } p \in A \text { and } y=f(p), \\ 0 & \text { otherwise. }\end{cases}
$$

Since the entire $A$ is contained in $G_{1}(t), \phi_{x}$ is an "approximately isometry" due to (8) and (9), and this implies by Lemma 6 and (10) also the statement about the density of $A_{x}$.

Remark. Theorem 9 immediately answers the question about the regularity of $\mathscr{H}_{\rho}^{n}$ on a $n$-rectifiable space of finite measure affirmatively. Indeed, the existence of $A_{x}$ shows that $\left.\liminf _{r \searrow 0}\left(\alpha(n) r^{n}\right)^{-1} \mathscr{H}_{\rho}^{n}\left(B_{\rho}(x, r) \cap X\right)\right) \geq 1$ holds $\mathscr{H}_{\rho}^{n}$-a.e., but the remaining inequality

$$
\bar{D}(X, x)=\limsup _{r \searrow 0}\left(\alpha(n) r^{n}\right)^{-1} \mathscr{H}_{\rho}^{n}\left(B_{\rho}(x, r) \cap X\right) \leq 1
$$

is true $\mathscr{H}_{\rho}^{n}$-a.e. in each space of finite measure (see [4, 2.10.19(5)]). Moreover, for rectifiable $(X, \rho)$ with $\mathscr{H}_{\rho}^{n}(X)<\infty$ the norm $\|\cdot\|_{x}$ constructed above is $\mathscr{H}_{\rho}^{n}$-a.e. unique up to linear isomorphisms; i.e., the "local norm" is a unique element of the Banach-Mazur compactum (see [9, Chapter 1]). Indeed, if $\bar{D}(X, x) \leq 1$ and if there are $\|\cdot\|_{x}, A_{x}, \phi_{x}$ and $\|\cdot\|_{x}^{\prime}, A_{x}^{\prime}, \phi_{x}^{\prime}$ fulfilling the conclusion of Theorem 9, then $\lim _{r \searrow 0}\left(\alpha(n) r^{n}\right)^{-1} \mathscr{L}^{n}\left(\phi_{x}\left(A_{x} \cap A_{x}^{\prime}\right)\right)=1$, and since $\phi_{x}^{\prime} \circ \phi_{x}^{-1}$ is differentiable a.e. on $\overline{\phi_{x}\left(A_{x} \cap A_{x}^{\prime}\right)}$, we see that a selected sequence $D\left(\phi_{x}^{\prime} \circ \phi_{x}^{-1}\right)\left(t_{m}\right), t_{m} \rightarrow 0$, converges to a linear isometry $i:\left(\mathbb{R}^{n},\|\cdot\|_{x}\right) \rightarrow\left(\mathbb{R}^{n},\|\cdot\|_{x}^{\prime}\right)$. This leads to the following definition.

Definition 10. Let $(X, \rho)$ be a metric space. Whenever there exists a norm $\|\cdot\|_{x}$, a set $A_{x} \subset X$, and a map $\phi_{x}$ as in the conclusion of Theorem 9 such that $\|\cdot\|_{x}$ is unique up to linear isomorphisms, we denote by $T N(X, x)$ the equivalence class of the Banach-Mazur compactum containing $\|\cdot\|_{x}$.

We finish the paper by showing a geometric aspect of $T N(X, x)$. For this we have to recall some notation. Let $\|\cdot\|$ be a norm on $\mathbb{R}^{n}$; then we denote

$$
\begin{aligned}
& \operatorname{vr}_{+}(\|\cdot\|)=\inf \left\{\mathscr{L}^{n}\left(B_{\|\cdot\|}(0,1)\right) / \mathscr{L}^{n}(E) \mid E \subset B_{\|\cdot\|}(0,1) \text { ellipsoid }\right\}, \\
& \operatorname{vr}_{-}(\|\cdot\|)=\sup \left\{\mathscr{L}^{n}\left(B_{\|\cdot\|}(0,1)\right) / \mathscr{L}^{n}(E) \mid E \supset B_{\|\cdot\|}(0,1) \text { ellipsoid }\right\},
\end{aligned}
$$


where $E \subset \mathbb{R}^{n}$ is an ellipsoid iff $E$ is a linear image of the Euclidian unit ball. A moment's reflection shows that $\mathbf{v r}_{ \pm}$is invariant under linear isomorphisms; consequently, we can also define $\operatorname{vr}_{ \pm}(T N(X, x))$. Due to a result of John (see [9, Chapter 3]), $\operatorname{vr}_{+}(\|\cdot\|) \leq n^{n / 2} \mathbf{v r}_{-}(\|\cdot\|)$ for any $\|\cdot\|$ on $\mathbb{R}^{n}$.

Further, we say that a (Borel regular outer) measure $\mu$ over the Banach space $C[0,1]$ is an ( $n$-dimensional) Kolmogoroff measure provided the following hold:

(1) For $A \subset C[0,1]$ and $f: A \rightarrow C[0,1]$ with $\operatorname{Lip}(f) \leq 1 \quad \mu(f(A)) \leq$ $\mu(A)$.

(2) $\mu(A)=1$ if $A \subset C[0,1]$ is isometric to the unit cube $[0,1]^{n}$ of the Euclidian $n$-dimensional space.

(We study $\mu$ over $C[0,1]$ since due to [2] any separable metric space isometrically embeds into $C[0,1]$.$) It was shown in [6] that there are a maximal and$ a minimal Kolmogoroff measure, defined for Borel $A \subset C[0,1]$ by

$$
\begin{gathered}
\bar{\mu}(A)=\inf \left\{\mathscr{L}^{n}(B) \mid B \subset \mathbb{R}^{n}, f: B \rightarrow C[0,1] 1-\text { Lip, and } f(B) \supset A\right\}, \\
\underline{\mu}(A)=\sup \left\{\sum_{i} \mathscr{L}^{n}\left(f_{i}\left(G_{i}\right)\right) \mid G_{i} \subset B\right. \text { disjoint Borel, } \\
\text { each } \left.f_{i}: G_{i} \rightarrow \mathbb{R}^{n} 1-\text { Lip }\right\} .
\end{gathered}
$$

In [6] it was also proved that $\bar{\mu}$ and $\mu$ agree on $n$-rectifiable subsets of Euclidian spaces. This result generalizes as follows.

Theorem 11. Let $M \subset C[0,1]$ be Borel and $\bar{\mu}(M)<\infty$. Then for any Borel subset $A \subset M$

$$
\begin{aligned}
& \underline{\mu}(A)=\int_{A} \operatorname{vr}_{-}(T N(M, x)) d \mathscr{K}_{\|\cdot\| \|}^{n}(x), \\
& \bar{\mu}(A)=\int_{A} \operatorname{vr}_{+}(T N(M, x)) d \mathscr{K}_{\|\cdot\| \|}^{n}(x),
\end{aligned}
$$

where $T N(M, x)$ is defined w.r.t. the metric subspace $M$ of $C[0,1]$.

Consequently, in this situation the following conditions are equivalent:

(1) $\underline{\mu}(M)=\mathscr{H}_{\|\cdot\|}^{n}(M)$,

(2) $\overline{\bar{\mu}}(\boldsymbol{M})=\mathscr{H}_{\|\cdot\|}^{n}(\boldsymbol{M})$,

(3) for $\mathscr{H}_{\|\cdot\|}^{n}$-a.e. $x \in M, T N(M, x)$ consists of all norms on $\mathbb{R}^{n}$ induced by an inner product.

Proof. First we determine the behaviour of $\underline{\mu}, \bar{\mu}$ on $n$-dimensional linear subspaces of $C[0,1]$. Since $\mu, \bar{\mu}$ are invariant under isometries, we can consider them on $\left(\mathbb{R}^{n},\|\cdot\|\right)$ and find constants $0 \leq c_{-} \leq c_{+} \leq \infty$ with $\underline{\mu}=c_{-} \mathscr{H}_{\|\cdot\|}^{n}$ and $\bar{\mu}=c_{+} \mathscr{X}_{\|\cdot\| \cdot}^{n}$. Let $E \subset B_{\|\cdot\|}(0,1)$ be any ellipsoid. Then the corresponding linear map $u$ with $u\left(B_{|\cdot|}(0,1)\right)=E$ is $(|\cdot|,\|\cdot\|)$-1-Lipschitzian. Hence,

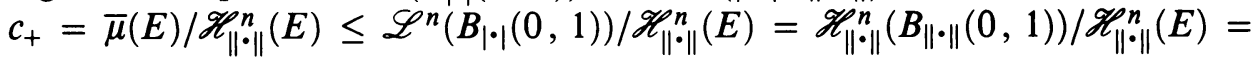
$\mathscr{L}^{n}\left(B_{\|\cdot\|}(0,1)\right) / \mathscr{L}^{n}(E)$. Consequently, $c_{+} \leq \operatorname{vr}_{+}(\|\cdot\|)$ and similarly $c_{-} \geq$ $\mathrm{vr}_{-}(\|\cdot\|)$ follows. Now we show $\mathrm{vr}_{+}(\|\cdot\|) \mathscr{K}_{\|} \cdot \|(f(A)) \leq \mathscr{L}^{n}(A)$ for any $A$ closed and 1-Lipschitzian $f:(A,|\cdot|) \rightarrow\left(\mathbb{R}^{n},\|\cdot\|\right)$; this already implies 
$c_{+} \geq \mathrm{vr}_{+}(\|\cdot\|)$. Since the target space is finite dimensional, $D f(x)$ exists for a.e. $x \in A$. But for such $x$ and the ellipsoid $E=D f(x)\left(B_{|\cdot|}(0,1)\right) \subset B_{\|\cdot\|}(0,1)$ we estimate $\mathscr{J}(M D(f, x))=\mathscr{J}(\|\cdot\| \circ D f(x))=\mathscr{X}_{\|\cdot\|}^{n}(E) / \mathscr{L}^{n}\left(B_{|\cdot|}(0,1)\right) \leq$ $\mathscr{K}_{\|\cdot\|}^{n}(E) / \mathscr{H}_{\|\cdot\|}^{n}\left(B_{\|\cdot\|}(0,1)\right) \leq 1 / v^{+}(\|\cdot\|)$, since $D f(x):\left(\mathbb{R}^{n}, M D(f, x)(\cdot)\right) \rightarrow$ $\left(\mathbb{R}^{n},\|\cdot\|\right)$ is an isometry. Now Corollary 8 gives the desired inequality. Finally, $c_{-} \leq \operatorname{vr}_{-}(\|\cdot\|)$ follows from $\mathscr{L}^{n}(f(G)) \leq \operatorname{vr}_{-}(\|\cdot\|) \mathscr{X}_{\|\cdot\|}^{n}(G)$ for all $G \subset \mathbb{R}^{n}$ open and $f:(G,\|\cdot\|) \rightarrow\left(\mathbb{R}^{n},|\cdot|\right)$ with $\operatorname{Lip}(f) \leq 1$. Again $D f$ exist a.e. on $G$ and it follows from the classical substitution rule already that $\mathscr{L}^{n}(f(G))=\mathscr{L}^{n}(f(R))$ where $R=\{x \in G \mid D f(x)$ injective $\}$. For any $x \in R, E=D f(x)^{-1}\left(B_{|\cdot|}(0,1)\right) \supset B_{\|\cdot\|}(0,1)$ is an ellipsoid; hence, the definition of $D f(x)$ yields: $\liminf _{r \backslash 0}\left(\alpha(n) r^{n}\right)^{-1} \mathscr{H}_{\|\cdot\|}^{n}\left(G \cap f^{-1}\left(B_{|\cdot|}(f(x), r)\right)\right) \geq$ $\liminf _{r \searrow 0}\left(\alpha(n) r^{n}\right)^{-1} \mathscr{H}_{\|\cdot\| \|}^{n}(x+r \cdot E)=\mathscr{H}_{\|\cdot\|}^{n}(E) / \mathscr{H}_{\|\cdot\| \|}^{n}\left(B_{\|\|}(0,1)\right) \geq 1 / v_{-}(\|\cdot\|)$. Therefore, the measure $\Phi(S)=\mathscr{H}_{\|\cdot\|}^{n}\left(f^{-1}(S) \cap G\right)$ has lower Lebesgue density greater or equal $1 / \mathrm{vr}_{-}(\|\cdot\|)$ a.e. on $f(G)$. Hence, $\mathscr{H}_{\|\cdot\|}^{n}(G)=\Phi(f(G)) \geq$ $1 / r_{-}(\|\cdot\|) \cdot \mathscr{L}^{n}(f(G))$ as required.

So we have derived the integral expressions for $\bar{\mu}$ and $\mu$ in case $M$ is linear. The rest of the proof is similar to considerations already carried out and will merely be outlined. Following the ideas of Theorem 7 and Corollary 8 we obtain that for $f: \mathbb{R}^{n} \rightarrow C[0,1]$ Lipschitzian, $\tilde{A} \subset \mathbb{R}^{n} \mathscr{L}^{n}$-measurable with $\mathscr{L}^{n}(\widetilde{A})<\infty$ and $g: C[0,1] \rightarrow[0, \infty] \bar{\mu}$-measurable

$$
\begin{aligned}
\int_{\widetilde{A}} g(f(x)) \mathrm{vr}_{+}(M D(f, x)) \mathscr{J}(M D(f, x)) d \mathscr{L}^{n}(x) \\
=\int_{C[0,1]} g(y) N(f \mid \widetilde{A}, y) d \bar{\mu}(y)
\end{aligned}
$$

where $\operatorname{vr}_{+}(M D(f, x)) \mathscr{J}(M D(f, x))=0$ for $\mathscr{J}(M D(f, x))=0$ due to the already mentioned result $\mathrm{vr}_{+}(\|\cdot\|) \leq n^{n / 2}$. We can suppose $M=f(\widetilde{M})$ for Borel $M \subset \mathbb{R}^{n}$ of finite measure. Then Corollary 8 ensures that $\bar{D}(M, f(x)) \leq$ 1 a.e. in $\widetilde{M} \cap \mathscr{M D}_{r}(f)$, and hence $M D(f, x) \in T N(M, f(x))$ for a.e. $x \in \widetilde{M} \cap \mathscr{M D}_{r}(f)$. Now, for given $A \subset M$ Borel we put $\widetilde{A}=\widetilde{M} \cap f^{-1}(A)$ and $g(y)=\min \left\{1, N(f \mid \widetilde{A}, y)^{-1}\right\}$. Applying (11), the foregoing remark about $T N(M, f(x))$, and Corollary 8 (ii) we can establish the conclusion for $\bar{\mu}$; we leave the details as well as the discussion concerning mensurability of the involved functions to the reader. For $\underline{\mu}$ the result is derived in the same way. The claimed equivalence is now obvious since $\|\cdot\|$ is induced by an inner product iff $B_{\|\cdot\|}(0,1)$ is an ellipsoid.

\section{REFERENCES}

1. N. Aronszajn, Differentiability of Lipschitz mappings between Banach spaces, Studia Math. 57 (1976), 147-190.

2. S. Banach and S. Mazur, Zur Theorie der linearen Dimension, Studia Math. 4 (1933), 100-112.

3. Yu. Burago and V. Zalgaller, Geometric inequalities, Grundlehren Math. Wiss., vol. 285, Springer, New York, 1988.

4. H. Federer, Geometric measure theory, Grundlehren Math. Wiss., vol. 153, Springer, New York, 1969. 
5. W. B. Johnson, J. Lindenstrauss, and G. Schechtman, Extension of Lipschitz maps into Banach spaces, Israel J. Math. 54 (1986), 129-138.

6. A. Kolmogoroff, Beiträge zur Maßtheorie, Math. Ann. 107 (1932), 351-366.

7. J. Lukeš, J. Malý, and L. Zajíček, Fine topological methods in real analysis and potential theory, Lecture Notes in Math., vol. 1189, Springer, New York, 1986.

8. P. Mattila, Hausdorff m-regular and rectifiable sets in $n$-space, Trans. Amer. Math. Soc. 107 (1975), 263-274.

9. G. Pisier, The volume of convex bodies and Banach space geometry, Cambridge Univ. Press, London and New York, 1989.

10. D. Preiss and J. Tišer, On Besicovitch $\frac{1}{2}$-problem, J. London Math. Soc. (2) 45 (1992), 279-287.

Institute of Applied Mathematics, Comenius University Bratislava, Mlynská dolina, Bratislava 842 15, Czechoslovakia

Current address: Institut für Mathematik, Johannes Kepler Universität, Altenbergerstrasse, A4040 Linz, Austria

E-mail address: kirchhei@mff.uniba.cs 